\title{
The effects of melatonin on the striatum
}

\author{
Sinem GERGIN ${ }^{1}$ (iD, Ozlem KIRAZLI ${ }^{1}$ (D), Hatice BORACI ${ }^{2}$ (D), Sercan Dogukan YILDIZ³ (D), Umit Suleyman SEHIRLI ${ }^{1}$ iD \\ ${ }^{1}$ Department of Anatomy, School of Medicine, Marmara University, Maltepe, Istanbul, Turkey. \\ ${ }^{2}$ Department of Anatomy, Institute of Health Sciences, Maltepe, Istanbul, Turkey. \\ ${ }^{3}$ Department of Anatomy, School of Dentistry, Kent University, Beyoglu, Istanbul, Turkey.
}

Corresponding Author: Sinem GERGIN

E-mail: drsinemgergin@gmail.com

Submitted: 09.03.2021 Accepted: 19.05.2021

\begin{abstract}
Objective: Some of the neurological diseases cause morphologic changes in the striatal neurons. Medial forebrain bundle (MFB) lesion is a commonly used method to produce a Parkinsonian model rat. Melatonin is a hormone which exerts a neuroprotective effect on the neurons. The aim of this study is to investigate the effect of melatonin on the dendritic morphology of striatal medium spiny neurons (MSNs) in rats with MFB lesion.

Materials and Methods: Twelve male Wistar albino rats were given saline injections into the MFB and divided into sedentary and treatment groups. The treatment group was administered a $10 \mathrm{mg} / \mathrm{kg}$ dose of melatonin intraperitoneally for 30 days. The lesion was confirmed histologically by Nissl staining. Golgi staining technique was applied to observe neuronal morphology. Neuronal structures were analysed from three-dimensional images by Neurolucida (MBF Bioscience) software.

Results: The MFB lesion caused a reduction in the total dendritic length and in the number of dendritic endings. The melatonin enhanced the number of dendritic endings compared to the sedentary group. The melatonin led to an increase in the total spine density, spine densities of thin and mushroom types.

Conclusion: Melatonin improved the dendritic degeneration due to MFB lesion.

Keywords: Dendritic spine, Melatonin, Golgi, Neurolucida
\end{abstract}

\section{INTRODUCTION}

The dendritic spines are small fingerlike protrusions that form excitatory synaptic connections. The spine morphology adjusts the stability and strength of the synapse [1]. The dynamic structural property of spine has a crucial role for synaptic plasticity [2]. Alterations in dendrite and spine morphology have been correlated with several neurological diseases $[3,4]$.

The spine morphology has a wide diversity with different head and neck sizes. The spine types are classified according to the ratio of head and neck diameter such as; thin $($ ratio $<2)$, mushroom (ratio $>2$ ), stubby $($ ratio $<1)$ and also branched (two heads and one neck) types $[1,5,6]$. The mushroom type is a stable and least dynamic spine. The mushroom spine is able to have strong synaptic connections and also essential for memory storage [7]. The thin type is a more dynamic spine that represents a small synapse and is significant for learning [8]. The stubby type is a dynamic and immature spine. The stubby type may be a general precursor of more mature spine form of mushroom and thin types [9]. The branched type has bifurcated head with a neck which is rarely seen [10]. The morphological alterations of the spines reflect significant knowledge about the neuronal plasticity.

Melatonin (N-acetyl-5-methoxytryptamine) is a hormone that is mainly synthesized in the pineal gland. Melatonin controls various physiological functions [11]. It also has anti-apoptotic, anti-tumor, and anti-oxidative properties [12,13]. Melatonin is highly lipophilic and passes across all morphological barriers (blood-brain barrier-BBB) and diffuses into all body fluids [14]. The disrupted BBB leads to the inflammatory reaction and neuronal cell damage that demonstrates the development or progression of central nervous system (CNS) diseases [15,16]. Melatonin reveales its beneficial effects in acute and chronic inflammatory processes $[17,18]$.

The recent techniques enable to observe a more detailed neuronal morphology. Three-dimensional computer-based microscopical systems provide fundamental opportunity to attain quantitative

How to cite this article: Gergin S, Kirazli O, Boraci H, Yildiz S D, Sehirli U S. The effects of melatonin on the striatum. Marmara Med J 2021 ; 34(3):234-240. doi: $10.5472 /$ marumj. 1012077 
information of neuron morphology and pathological changes. The Neurolucida is a commonly used software that facilitates different laboratories to standardize the parameters of somatodendritic morphological data [19].

The striatum is a part of the basal ganglia. It contains medium spiny neurons (MSNs) and a few interneurons [20]. Neurodegenerative diseases like Parkinson's disease lead to degeneration of the striatal neurons. In experimental models, medial forebrain bundle (MFB) is a common target to induce 6-hydroxydopamine (6-OHDA) lesion to produce a Parkinsonian model rat. Many electrophysiological studies showed that the 6-OHDA lesion in the nigrostriatal pathway led to functional loss of striatal neurons and interneurons. After the formation of the lesion, the inhibitory effect of GABAergic interneurons against the MSNs increased [21]. The impairment of striatal neurons showed motor, cognitive and behavioral symptoms that were correlated with the functional and morphological deterioration [22].

In view of high technology, spine morphology alterations reflect different functions. In the current study, we aimed to investigate the effects of melatonin on the dendrite and spine morphology of striatal neurons after minimal MFB lesion.

\section{MATERIALS and METHODS}

\section{Animals and Groups}

Twelve male Wistar albino rats (weighing 250-300 g, 9 weeks of age) were used in this study. The rats were obtained from Marmara University Experimental Animal Research Center. All experimental procedures were approved by the Ethics Committee for Animal Experimentation of Marmara University (42.2021mar, 3.15.2021).

All animals were maintained in cages ( 2 rats per cage) in a room with controlled temperature $\left(20^{\circ} \mathrm{C} \pm 2^{\circ} \mathrm{C}\right)$ under a 12 -h light/ dark cycle with standard rat feed and water supply. The rats were randomly divided into 2 groups (6 in each group); MFB injection + sedentary and MFB injection + melatonin.

\section{Animal Model}

Stereotaxic surgery was performed to inject saline into the MFB. A mixture of ketamine $(100 \mathrm{mg} / \mathrm{kg})$ and xylazine $(50 \mathrm{mg} / \mathrm{kg})$ were administered intraperitoneally to anaesthetize the rats. The coordinates of right MFB were the rostral AP (anterior posterior): $-2.1, \mathrm{~L}: 2.0, \mathrm{~V}:-7.8$ (in $\mathrm{mm}$ ) and caudal AP: -4.3 , L: $1.5 \mathrm{~V}:-7.8$ [23]. A total of $8 \mu \mathrm{l} 0.9 \%$ saline were injected at a rate of $1 \mu \mathrm{l} / \mathrm{min}$ using a Hamilton syringe (Stoelting/10 $\mu \mathrm{l}$ ) and an infusion pump (KdScientific, MA, USA). At the end of the injection, the syringe was left in the MFB for 5 minutes. Then, it was pulled back slowly. After the procedure, $5 \mathrm{ml}$ of saline $(0.9 \%$ $\mathrm{NaCl}$ ) was injected subcutaneously.

\section{Melatonin Preparation}

Melatonin (Sigma, St Louis, MO, USA, M5250) was dissolved in ethanol, and diluted in saline solution (5:95). The final concentration of the melatonin was $10 \mathrm{mg} / \mathrm{ml}$. The melatonin was administered intraperitoneally at a dose of $10 \mathrm{mg} / \mathrm{kg}$ at the same day time (for 30 days). The drug was prepared freshly before the injection.

\section{Tissue Collection and Golgi Staining}

The rats were killed by transcardial perfusion with $0.9 \%$ saline followed by $4 \%$ paraformaldehyde (PFA) (Merck, Darmstadt, Germany), (0.1 M phosphate buffer, $\mathrm{pH}$ 7.4) after deeply anesthetized with ketamine (100 mg/kg, intraperitoneally). The brains were removed and sectioned for staining. In order to determine the accuracy of the MFB injection site, Nissl staining was performed and examined through a light microscope (Figure 1).

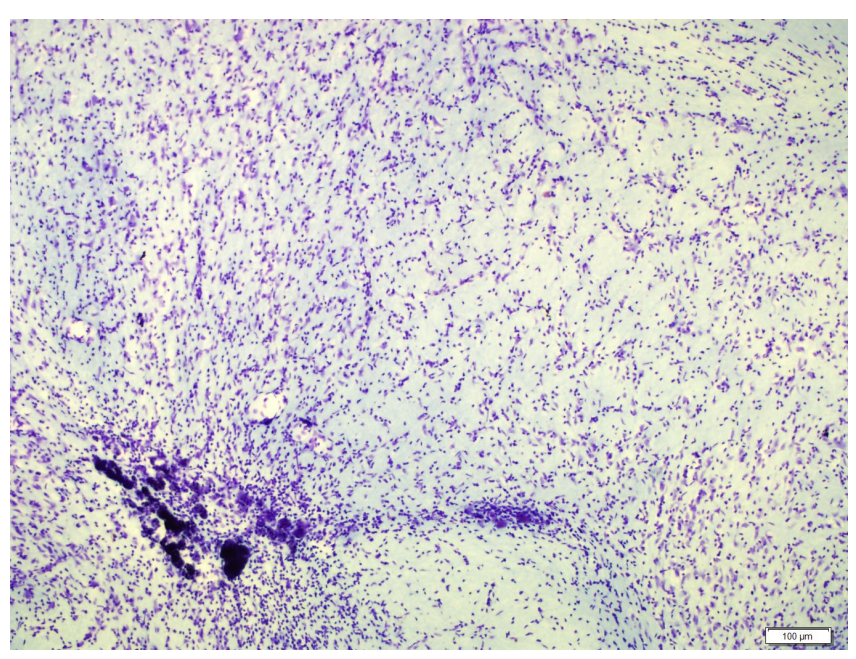

Figure 1. Examination of the MFB injection site by Nissl staining using a $10 \mathrm{X}$ objective under a light microscope.

Golgi-cox staining technique was performed to observe the morphological features of the dendrite and the spine in detail [1]. FD Rapid GolgiStain Kit ${ }^{\mathrm{TM}}$ (FD NeuroTechnologies Inc. Elliot City, MD, USA) which is a commercially available Golgi staining kit was used according to the instructions. The kit contains standard A, B, C, D, E solutions. After perfusion, the brain tissues were rinsed with double distilled water then they were immersed in the impregnation solution, made by mixing equal volumes of solutions $\mathrm{A}$ and $\mathrm{B}$ (mercuric chloride, potassium dichromate and potassium chromate). Then, it was stored at room temperature for 3 weeks in the dark. The brain tissues were replaced in the impregnation solution (A and B again) after 24 hours then tissues were transferred into solution $\mathrm{C}$ and stored at room temperature in the dark for one week before slicing. The brain sections were cut in $100 \mu \mathrm{m}$ on a cryostat (Leica Biosystems, CM 1950, USA) at $20^{\circ} \mathrm{C}$. Each section was transferred with a glass specimen and mounted on gelatin-coated microscope slides with solution $\mathrm{C}$ and stored at room temperature in the dark until they were dried out. The slices were rinsed in double distilled water and then placed in a mixture consisting of 1 fraction solution D, 
1 fraction solution $\mathrm{E}$ and 2 fractions of double distilled water for 10 minutes. After sections were rinsed in double distilled water, slices were dehydrated in series of 50\%, 75\%, 95\% and $100 \%$ ethanol respectively and lastly cleared in xylene and cover-slipped by the aid of permount.

\section{Light Microscopic Analysis}

Golgi-stained sections were investigated using an Olympus BX51 microscope and Q Imaging Retiga-2000R camera. Neuronal structures were examined from three-dimensional images using Neurolucida software (MBF Bioscience, Williston, WT, USA). MSN selection and classification of neuronal features were made intently by an observer blinded to the animal groups. For each group, 2 neurons on the lesion and contralateral sides were analysed giving a total of 48 neurons and 96 dendrites. For quantitative analysis, neuron body and dendrites were drawn using a $60 \mathrm{X}$ objective. After, the dendritic spines of the first $10 \mu \mathrm{m}$ length from the branching area on the secondary dendrites (nearest branch point) were marked. Then, the spines were classified according to the dendritic spine head and neck ratio by a $100 \mathrm{X}$ objective. Sholl analysis was performed for each neuron to maintain morphologic parameters per $10 \mu \mathrm{m}$ in diameter concentric circles (Figure 2 ). The dendritic morphometric analysis was performed by Neurolucida Explorer. Total dendritic length (total length of primary and secondary dendritic branches), number of dendritic branches (total number of primary and secondary dendritic segments), nodes (total dendritic bifurcation points), endings (total dendritic termination points), total spine density (spines $/ 10 \mu \mathrm{m}$ dendrite), morphologic classification of spines (thin, mushroom, stubby, branched) and densities were analized.

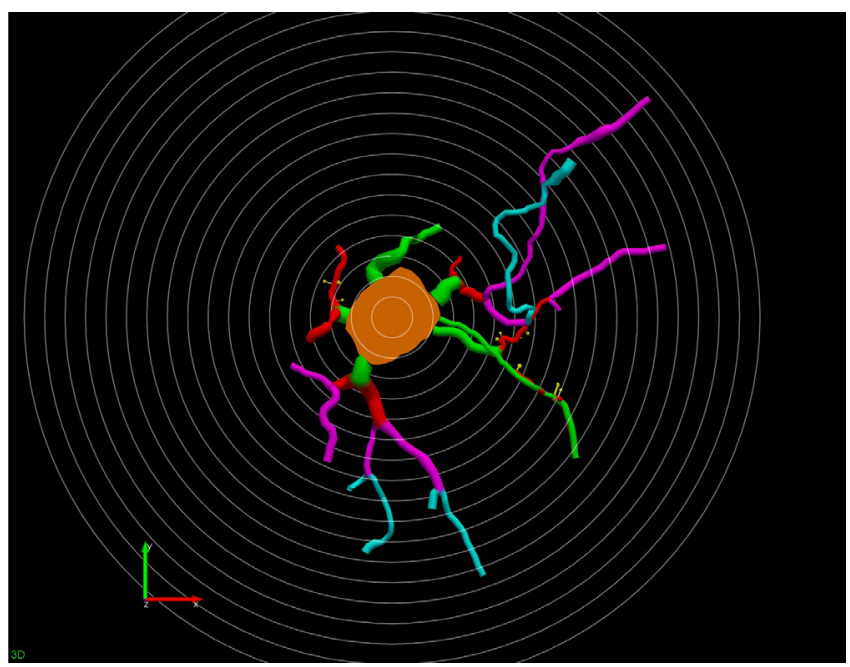

Figure 2. 3D reconstruction of the Sholl analysis of a MSN in the melatonin group. (Scale bar $10 \mu \mathrm{m}$ ). The neuron body is shown in orange. The dendritic branches of neuron are represented with different colors (green-primary, red-secondary, pink-tertiary, blue - quaternary). The spines are shown in yellow arrows on secondary branches of dendrites.

\section{Statistical Analysis}

The GraphPad Prism 6 software was used for the analysis of the data. The normal distribution of data was evaluated by Kolmogorov-Smirnov test. Based on this, whenever the data pass the normality test, parametric Sample t-test was performed. When the data did not pass the normality test, non-parametric Mann-Whitney $U$ test was performed. Data in the text and figures were shown as mean \pm standard error of mean (SEM). The data was represented as "p value". A value of $p<0.05$ was considered statistically significant.

\section{RESULTS}

\section{Morphological Analyses of MSNs}

The dendritic branching pattern and spine morphology were observed by using Neurolucida software on MSNs in the striatum. The MFB lesion resulted in the reduction of the total dendritic length $(181.1 \pm 28.1 \mu \mathrm{m}, \mathrm{n}=6)$ compared to the contralateral side $(366.3 \pm 58.7 \mu \mathrm{m}, \mathrm{n}=6)$ of the sedentary group $(\mathrm{t}=1.40, \mathrm{df}=10$; $\mathrm{p}=0.0174$; Figure 3). The MFB lesion led to a significant reduction in the total dendritic endings $(6 \pm 0.5, \mathrm{n}=6)$ compared to the contralateral side $(9 \pm 0.8, \mathrm{n}=6)$ of the sedentary group $(\mathrm{t}=2.98$, $\mathrm{df}=10 ; \mathrm{p}=0.0137$, Figure 4 ). The total dendritic length on the lesion side did not show any significant difference between the melatonin $(244 \pm 34.9 \mu \mathrm{m}, \mathrm{n}=6)$ and sedentary $(181.1 \pm 28.1 \mu \mathrm{m}, \mathrm{n}=6)$ groups $(\mathrm{p}=0.19)$. The melatonin $(8.3 \pm 0.8, \mathrm{n}=6)$ enhanced the number of dendritic endings compared to the sedentary group $(2.4 \pm 0.6$, $n=6),(t=5.81, d f=10 ; p=0.0002$, Figure 5). Moreover, the number of dendritic branches and nodes on the lesion side did not show statistically significant difference either between the contralateral sides of each group or melatonin and sedentary groups (branches; $\mathrm{p}=0.93$, nodes; $\mathrm{p}=0.99$ ). The dendritic complexity on the lesion side did not reveal a significant difference either between the melatonin and sedentary groups $(\mathrm{p}=0.88)$ or contralateral sides of each group $(\mathrm{p}=0.97)$.

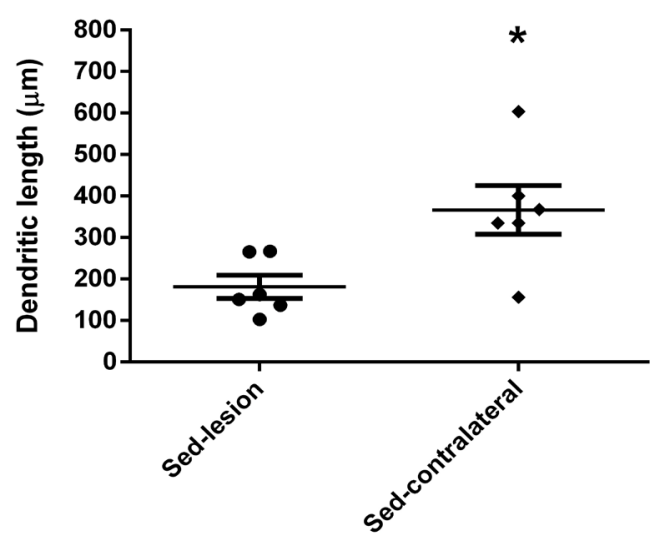

Figure 3. The effects of MFB lesion on total dendritic length ( $\mu \mathrm{m})$. The lesion side of the number of the total dendritic length was significantly lower compared to the contralateral side of sedentary group $(p=0.0174$, $\left.{ }^{*} p<0.05\right)$. 


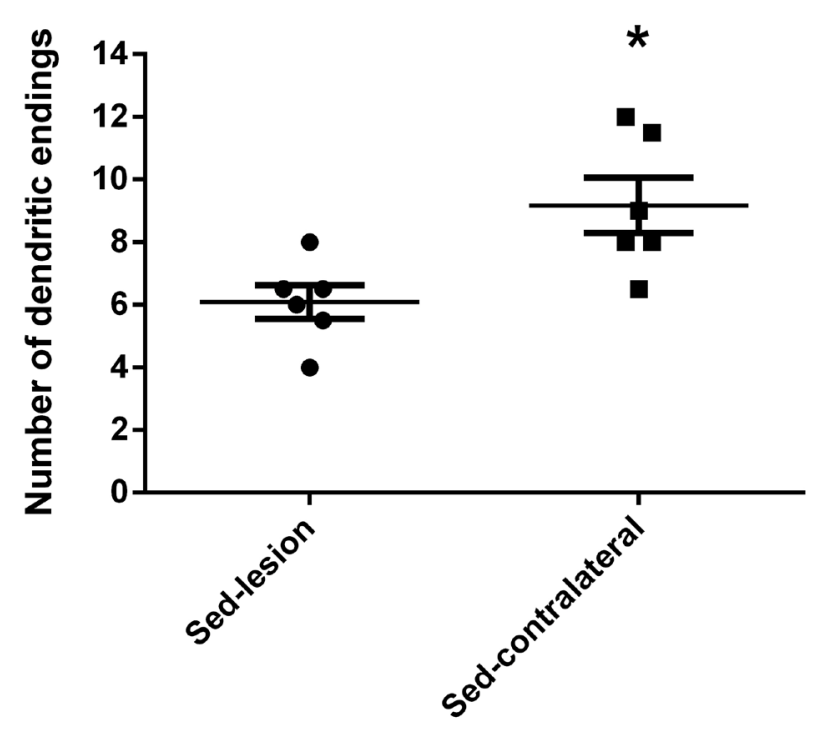

Figure 4. The effects of MFB lesion on number of dendritic endings. The lesion side of the number of the dendritic endings was significantly lower compared to the contralateral side of sedentary group $\left(p=0.0137,{ }^{*} p<0.05\right)$

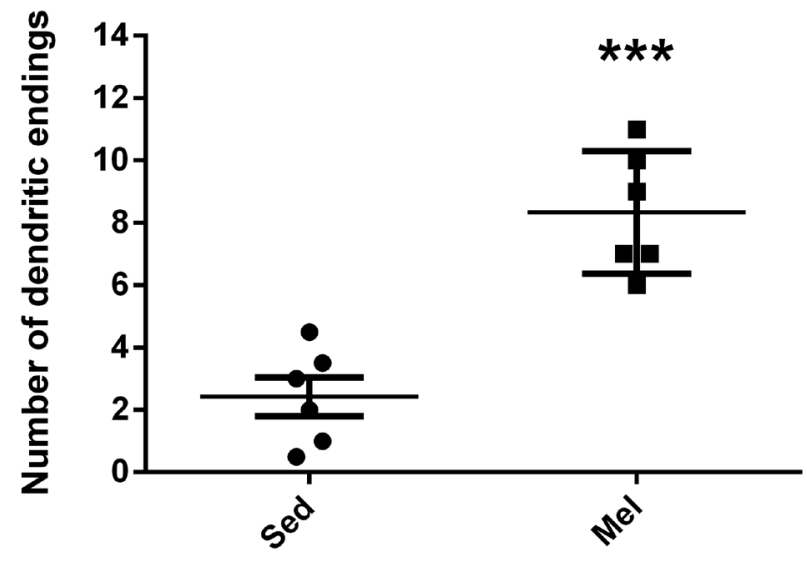

Figure 5. The effect of melatonin on number of dendritic endings. The melatonin increased the number of dendritic endings compared to the sedentary group $\left(p=0.0002,{ }^{* *} p<0.001\right)$.

The effect of melatonin $(7.4 \pm 1.1$ spines $/ 10 \mu \mathrm{m}, \mathrm{n}=6)$ increased the total spine density compared to the sedentary group $(3 \pm$ 1.2 spines $/ 10 \mu \mathrm{m}, \mathrm{n}=6)(\mathrm{t}=2.70, \mathrm{df}=10 ; \mathrm{p}=0.0223$; Figure 6$)$. The spine density of thin type was increased by the melatonin $(3.1 \pm$ 0.6 thin spines $/ 10 \mu \mathrm{m})$ compared to the sedentary group $(1.1 \pm 0.1$ thin spines $/ 10 \mu \mathrm{m}, \mathrm{n}=6)(\mathrm{t}=3.2, \mathrm{df}=10 ; \mathrm{p}=0.0092$; Figure 7$)$. The spine density of mushroom type was enhanced in the melatonin $(3.2 \pm 0.6$ mushroom spines $/ 10 \mu \mathrm{m}, \mathrm{n}=6)$ group compared to the sedentary group $(1.1 \pm 0.5$ mushroom spines $/ 10 \mu \mathrm{m}),(\mathrm{t}=2.25$, $\mathrm{df}=10 ; \mathrm{p}=0.0303$; Figure 8 ). The spine densities of stubby and branched types did not show statistically significant difference either between melatonin and sedentary groups or contralateral sides of each group (stubby; $\mathrm{p}=0.07$, branched; $\mathrm{p}=0.35$ ).

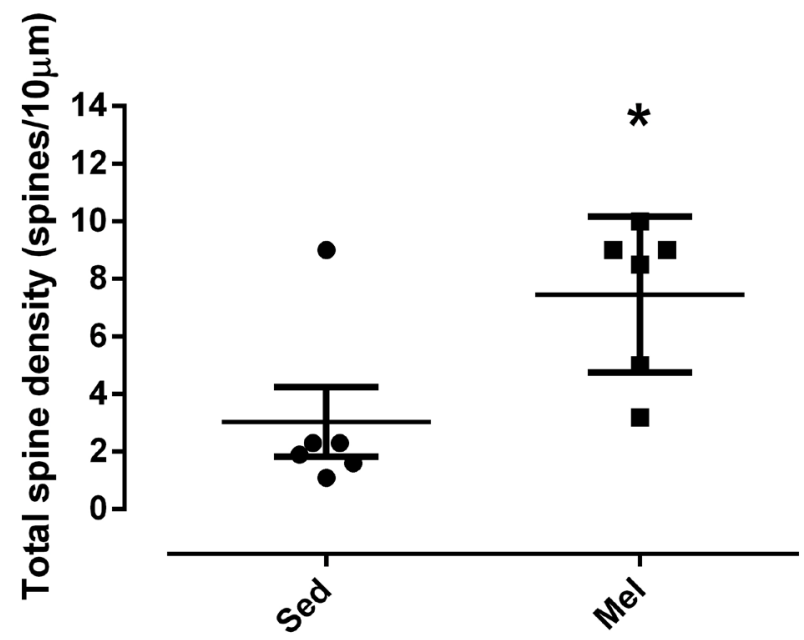

Figure 6. The effects of MFB lesion and melatonin on total spine density (spines/10 $\mu \mathrm{m})$. The melatonin group increased the total spine density compared to the sedentary groups $\left(p=0.0223,{ }^{*} p<0.05\right)$.

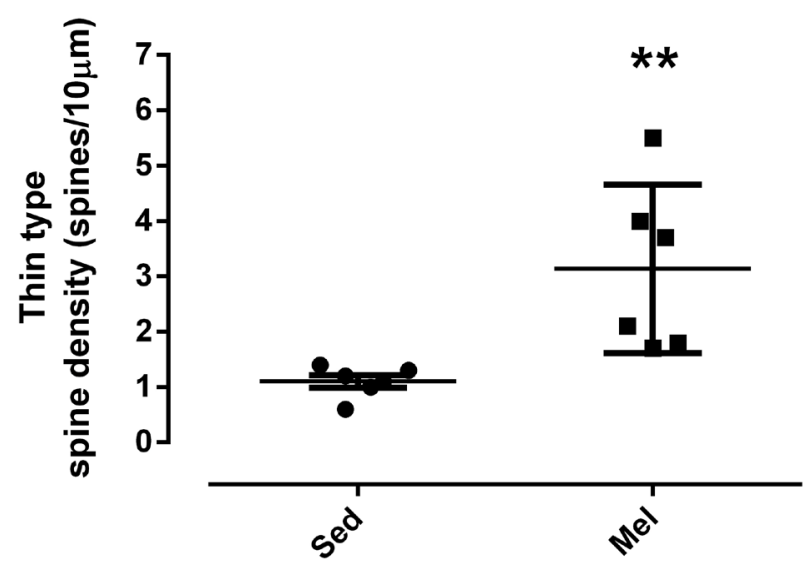

Figure 7. The effects of MFB lesion and melatonin on spine density of thin

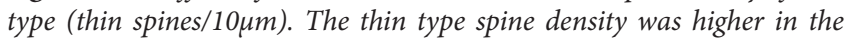
melatonin group compared to the sedentary group $\left(p=0.0092,{ }^{* *} p<0.01\right)$. 


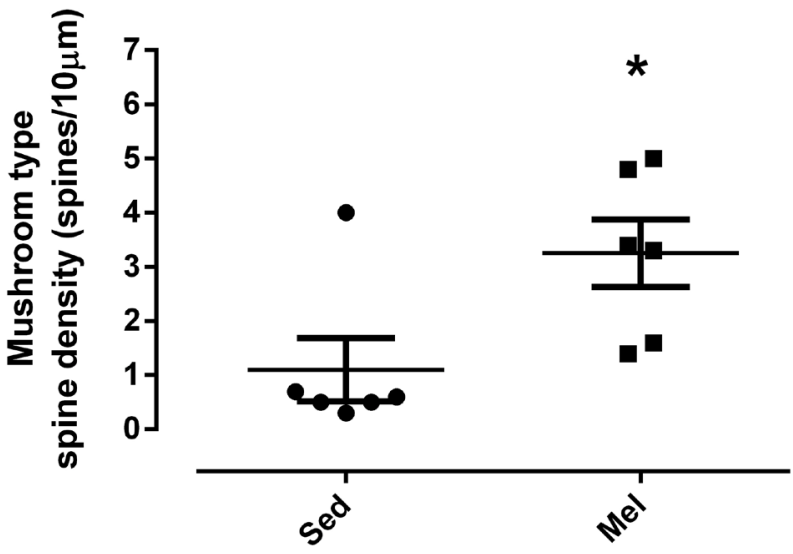

Figure 8. The effects of MFB lesion and melatonin on mushroom type spine density (mushroom spines/10 $\mu \mathrm{m}$ ). The mushroom type spine density was higher in the melatonin group compared to the sedentary group $\left(p=0.0303,{ }^{*} p<0.05\right.$

\section{DISCUSSION}

The potential effects of melatonin on the lesioned striatal neurons were analysed in this study. The MFB lesion led to a reduction of the total dendritic length and the number of dendritic endings compared to the contralateral side of the sedentary group. The melatonin improved the number of dendritic endings compared to the sedentary group. The number of dendritic branches and the nodes were not affected by MFB lesion and also melatonin did not cause any significant effect on these structures. Therefore, the dendritic complexity did not show any significant difference between the melatonin and sedentary groups. The administration of melatonin increased the total spine density and the spine densities of thin and mushroom types.

The dendritic branching pattern of MSNs was affected by many abnormal conditions [24,25]. The cerebral hypoxic ischemia revealed the impaired dendritic arbors such as; branches, nodes, endings and spines in an animal model of MSNs [26]. Some psychostimulant drugs have been shown to increase the dendritic branching in animal models of drug abuse [27]. In contrast, the 6-OHDA and 1-methyl-4-phenyl-1,2,3,6-tetrahydropyridine (MPTP) injected Parkinsonian models, there was a decrease in the dendritic length and spine density that was correlated with the loss of dopamine $[28,29]$. The recent literature showed that dopamine secretion regulates the formation and the density of dendritic spines in the striatum [30]. A previous study demonstrated the decrease of the spine densities of thin and mushroom types and an increase of the spine density of stubby type in MSNs following 6-OHDA lesion [8]. This study showed that a minimal trauma to the MFB may cause a limited degree of damage by affecting the dendrite. This information should be kept in mind during planning the Parkinson's disease model studies.
Exogenous melatonin is an important endocrine hormone due to its BBB penetration and easy tolerability without any side effects. Melatonin regulates the expression of neurotrophins and also ameliorates the motor and behavioral functions [31].

Melatonin has antioxidative and neuroprotective effects. It has been suggested as a beneficial antioxidant in the treatment of central nervous system disorders such as brain injuries, Parkinson's disease, Huntington and Alzheimer's disease $[32,33]$. The studies reported that melatonin had an effect on oxidative stress by increasing antioxidant enzymes and mitochondrial complex-I [34,35]. A study reported that melatonin improved the reduction in the spine density of MSN after MPTP injection [36]. The literature suggested that several types of spines reflect different synaptic functions [9,37]. In the present study, melatonin improved the dendritic endings, total spine density and spine densities of thin and mushroom types. The mushroom type has more synaptic ability because of containing excess glutamatergic receptors compared to the other types $[7,38]$. Thin spines are more dynamic form for synaptic plasticity that are also associated with the cognitive function $[8,39]$. The neuronal plasticity occurs in two ways which are functional and structural. In addition, the neuronal plasticity involves alterations in the dendritic spines rather than dendrites in the mature brain [40]. In our study, the structural changes observed in melatonin group were considered as plasticity. The increases of thin and mushroom spine types in the melatonin group represented a more stable morphological form of the spine. This is probably correlated with its synaptic ability. The total dendritic length in the striatum was positively affected by melatonin. Moreover, there was no statistically significant difference in the spine densities of stubby and branched types between the groups. We put forth that the melatonin was more effective on the spine density compared to the dendritic arborization.

Our study showed the effects of MFB lesion and the effects of melatonin on the striatal neurons. A minimal MFB lesion influenced the dendritic parameters rather than the spines. The melatonin ameliorated the morphologic features of dendrites and spines. Melatonin has beneficial effect on the spine density. There is conflicting data in the literature regarding the functions of different spine types $[41,42]$. The data provided from our study will also contribute to the understanding of the morphology and function of dendrites and spines in the striatum.

\section{Compliance with Ethical Standards}

Ethical Approval: The study protocol was approved by the Animal Experimentation Ethical Committee of Marmara University (approval number: 42.2021.mar).

Financial Support: No special funding was obtained.

Conflict of Interest Statement: There is no conflict of interest.

Author Contributions: : S.G., U.S.S. Finding the topic, performing the experiment, analysis and writing the manuscript. O.K. Performed the Golgi staining and statistical analysis. H.B.Performed the Golgi staining. S.D.Y. Contributed to the 
analysis at Neurolucida software. All authors have read and approved the final version of the article.

\section{REFERENCES}

[1] Arellano JI, Benavides-Piccione R, Defelipe J, Yuste R. Ultrastructure of dendritic spines: correlation between synaptic and spine morphologies. Front Neurosci 2007; 1:13143. doi: 10.3389/neuro.01.1.1.010.2007.

[2] Yuste R. Dendritic spines. Cambridge, Massachusetts: MIT Press, 2010: 162.

[3] Herms J, Dorostkar MM. Dendritic spine pathology in neurodegenerative diseases. Annu Rev Pathol 2016; 11:22150. doi: 10.1146/annurev-pathol-012.615.044216.

[4] Solis O, Limón DI, Flores-Hernández J, Flores G. Alterations in dendritic morphology of the prefrontal cortical and striatum neurons in the unilateral 6-OHDA-rat model of Parkinson's disease. Synapse 2007; 61:450-8. doi: 10.1002/syn.20381.

[5] Peters A, Kaiserman-Abramof IR. The small pyramidal neuron of the rat cerebral cortex. The perikaryon, dendrites and spines. Am J Anat 1970; 127:321-55. doi: 10.1002/ aja.100.127.0402.

[6] Spires TL, Grote HE, Garry S, et al. Dendritic spine pathology and deficits in experience-dependent dendritic plasticity in R6/1 Huntington's disease transgenic mice. Eur J Neurosci 2004; 19:2799-2807. doi: 10.1111/j.0953-816X.2004.03374.x.

[7] Grutzendler J, Kasthuri N, Gan WB. Long-term dendritic spine stability in the adult cortex. Nature 2002; 420:812-6. doi: 10.1038/nature01276.

[8] Suarez LM, Solis O, Aguado C, Lujan R, Moratalla R. L-DOPA oppositely regulates synaptic strength and spine morphology in D1 and D2 striatal projection neurons in dyskinesia. Cereb Cortex 2016; 26:4253-64. doi: 10.1093/cercor/bhw263.

[9] Bourne J, Harris KM. Do thin spines learn to be mushroom spines that remember? Curr Opin Neurobiol 2007; 17:381-6. doi: 10.1016/j.conb.2007.04.009.

[10] Comery TA, Stamoudis CX, Irwin SA, Greenough WT. Increased density of multiple-head dendritic spines on medium-sized spiny neurons of the striatum in rats reared in a complex environment. Neurobiol Learn Mem 1996; 66:9396. doi: 10.1006/nlme.1996.0049.

[11] Tordjman S, Chokron S, Delorme R, et al. Melatonin: Pharmacology, Functions and Therapeutic Benefits. Curr Neuropharmacol 2017; 15:434-43. doi: 10.2174/1570159X14 6.661.61228122115.

[12] Reiter RJ, Mayo JC, Tan DX, Sainz RM, Alatorre-Jimenez M, Qin L. Melatonin as an antioxidant: under promises but over delivers. J Pineal Res 2016; 61:253-78. doi: 10.1111/jpi.12360.

[13] Wang $X$. The antiapoptotic activity of melatonin in neurodegenerative diseases. CNS Neurosci Ther 2009; 15:34557. doi: 10.1111/j.1755-5949.2009.00105.x.

[14] Pandi-Perumal SR, BaHammam AS, Brown GM, et al. . Melatonin antioxidative defense: therapeutical implications for aging and neurodegenerative processes. Neurotox Res 2013; 23:267-300. doi: 10.1007/s12640.012.9337-4.
[15] Stolp HB, Dziegielewska KM, Ek CJ, Potter AM, Saunders NR. Long-term changes in blood-brain barrier permeability and white matter following prolonged systemic inflammation in early development in the rat. Eur J Neurosci 2005; 22:28052816. doi: 10.1111/j.1460-9568.2005.04483.x.

[16] Ozdemir D, Uysal N, Gonenc S, et al. Effect of melatonin on brain oxidative damage induced by traumatic brain injury in immature rats. Physiol Res 2005; 54:631-7. doi: 10.3390/ ijms19051539.

[17] Polimeni G, Esposito E, Bevelacqua V, Guarneri C, Cuzzocrea S. Role of melatonin supplementation in neurodegenerative disorders. Front Biosci (Landmark Ed) 2014; 19:429-446. doi: 10.2741/4217. PMID: 24389194.

[18] Hardeland R. Melatonin and inflammation-Story of a doubleedged blade. J Pineal Res 2018; 65:e12525. doi: 10.1111/ jpi.12525.

[19] Bicanic I, Hladnik A, Petanjek Z. A quantitative Golgi study of dendritic morphology in the mice striatal medium spiny neurons. Front Neuroanat 2017; 11:37. doi: 10.3389/ fnana.2017.00037.

[20] Tepper JM, Abercrombie ED, Bolam JP. Basal ganglia macrocircuits. Prog Brain Res 2007; 160:3-7. doi: 10.1016/ S0079-6123(06)60001-0.

[21] Gittis AH, Nelson AB, Thwin MT, Palop JJ, Kreitzer AC. Distinct roles of GABAergic interneurons in the regulation of striatal output pathways. J Neurosci 2010; 30:2223-34. doi: 10.1523/JNEUROSCI.4870-09.2010.

[22] O'Callaghan C, Bertoux M, Hornberger M. Beyond and below the cortex: the contribution of striatal dysfunction to cognition and behaviour in neurodegeneration. J Neurol Neurosurg Psychiatry 2014; 85:371-8. doi: 10.1136/jnnp-2012-304558.

[23] Paxinos G, Watson C. The rat brain in stereotaxic coordinates. 6th ed. USA: Academic Press, 2007.

[24] Bicanic I, Hladnik A, Petanjek Z. A Quantitative Golgi Study of Dendritic Morphology in the Mice Striatal Medium Spiny Neurons. Front Neuroanat. 2017; 11:37. doi:10.3389/ fnana.2017.00037

[25] Kulkarni VA, Firestein BL. The dendritic tree and brain disorders. Mol Cell Neurosci 2012; 50:10-20. doi: 10.1016/j. mcn.2012.03.005.

[26] McClendon E, Chen K, Gong X, et al. Prenatal cerebral ischemia triggers dysmaturation of caudate projection neurons. Ann Neurol 2014; 75:508-24. doi: 10.1002/ana.24100.

[27] Robinson TE, Kolb B. Structural plasticity associated with exposure to drugs of abuse. Neuropharmacology 2004; 47:3346. doi: 10.1016/j.neuropharm.2004.06.025.

[28] Villalba RM, Smith Y. Differential structural plasticity of corticostriatal and thalamostriatal axo-spinous synapses in MPTP-treated Parkinsonian monkeys. J Comp Neurol 2011; 519:989-1005. doi: 10.1002/cne.22563.

[29] Walker RH, Moore C, Davies G, Dirling LB, Koch RJ, Meshul CK. Effects of subthalamic nucleus lesions and stimulation upon corticostriatal afferents in the 6-hydroxydopaminelesioned rat. PLoS One 2012; 7:e32919. doi: 10.1371/journal. pone.0032919. 
[30] Soderstrom KE, O’Malley JA, Levine ND, Sortwell CE, Collier TJ, Steece-Collier K. Impact of dendritic spine preservation in medium spiny neurons on dopamine graft efficacy and the expression of dyskinesias in parkinsonian rats. Eur J Neurosci 2010; 31:478-90. doi: 10.1111/j.1460-9568.2010.07077.x.

[31] Mack JM, Schamne MG, Sampaio TB, et al. Melatoninergic system in Parkinson's disease: from neuroprotection to the management of motor and nonmotor symptoms. Oxid Med Cell Longev 2016; 2016:3472032. doi: 10.1155/2016/3472032.

[32] Osier N, McGreevy E, Pham L, et al. Melatonin as a therapy for traumatic brain injury: A review of published evidence. Int J Mol Sci 2018; 19:1539. doi: 10.3390/ijms19051539.

[33] Wongprayoon P, Govitrapong P. Melatonin as a mitochondrial protector in neurodegenerative diseases. Cell Mol Life Sci 2017; 74:3999-4014. doi: 10.1007/s00018.017.2614-x.

[34] Reiter RJ, Mayo JC, Tan DX, Sainz RM, Alatorre-Jimenez M, Qin L. Melatonin as an antioxidant: under promises but over delivers. J Pineal Res 2016; 61:253-78. doi: 10.1111/jpi.12360.

[35] Saravanan KS, Sindhu KM, Mohanakumar KP. Melatonin protects against rotenone-induced oxidative stress in a hemiparkinsonian rat model. J Pineal Res 2007; 42:247-53. doi: 10.1111/j.1600-079X.2006.00412.x.

[36] Naskar A, Manivasagam T, Chakraborty J, et al. Melatonin synergizes with low doses of L-DOPA to improve dendritic spine density in the mouse striatum in experimental Parkinsonism. J Pineal Res 2013; 55:304-12. doi:10.1111/ jpi.12076.
[37] Chidambaram SB, Rathipriya AG, Bolla SR, et al. Dendritic spines: Revisiting the physiological role. Prog Neuropsychopharmacol Biol Psychiatry 2019; 92:161-93. doi: 10.1016/j.pnpbp.2019.01.005.

[38] Bello-Medina PC, Flores G, Quirarte GL, McGaugh JL, Prado Alcalá RA. Mushroom spine dynamics in medium spiny neurons of dorsal striatum associated with memory of moderate and intense training. Proc Natl Acad Sci U S A. 2016;113:E6516-E6525. doi:10.1073/pnas.161.368.0113.

[39] Dumitriu D, Hao J, Hara Y, et al. Selective changes in thin spine density and morphology in monkey prefrontal cortex correlate with aging-related cognitive impairment. J Neurosci 2010; 30:7507-7515. doi: 10.1523/JNEUROSCI.6410-09.2010.

[40] Krohmer RW, Jurkovic J. Neuronal plasticity in the forebrain of the male red-sided garter snake: Effect of season, low temperature dormancy, and hormonal status on dendritic spine density. Physiol Behav 2020; 215:112789. doi: 10.1016/j. physbeh.2019.112789.

[41] Bourne J, Harris KM. Do thin spines learn to be mushroom spines that remember? Curr Opin Neurobiol 2007;17:381-6. doi:10.1016/j.conb.2007.04.009.

[42] Bello-Medina PC, Flores G, Quirarte GL, McGaugh JL, Prado Alcalá RA. Mushroom spine dynamics in medium spiny neurons of dorsal striatum associated with memory of moderate and intense training. Proc Natl Acad Sci U S A. 2016;113:E6516-E6525. doi:10.1073/pnas.161.368.0113. 